\title{
Realidade virtual na reabilitação motora da Doença de Parkinson: revisão integrativa
}

\author{
Virtual reality in motor rehabilitation of Parkinson's \\ Disease: integrative review
}

\author{
Realidad virtual en la rehabilitación motora de la \\ Enfermedad de Parkinson: revisión integrativa
}

\author{
Haylane Nunes da Conceição' ${ }^{1}$ Joelson da Silva Medeiros², Daniela \\ Farias de Carvalho ${ }^{3}$
}

\begin{abstract}
1.Discente do curso Bacharelado em Fisioterapia, Centro Universitário de Ciências e Tecnologia do Maranhão, UNIFACEMA. Caxias-MA, Brasil. Orcid: https://orcid.org/0000-0001-9508-9615

2.Fisioterapeuta, Mestre em Engenharia Biomédica, Centro Universitário de Ciências e Tecnologia do Maranhão, UNIFACEMA. Caxias-MA, Brasil. Orcid: https://orcid.org/0000-0002-6339-2916

3.Fisioterapeuta, Mestranda em Ciências Biomédicas, Docente do curso Bacharelado em Fisioterapia, Centro Universitário de Ciências e Tecnologia do Maranhão, UNIFACEMA. Caxias-MA, Brasil. Orcid: https://orcid.org/0000-0003-3499-064X
\end{abstract}

\begin{abstract}
Resumo
Introdução. A realidade virtual é uma ferramenta tecnológica utilizada no protocolo fisioterapêutico de reabilitação, sendo uma opção de tratamento para os indivíduos acometidos pela Doença de Parkinson (DP). Objetivo. Abordar as contribuições da realidade virtual na reabilitação das alterações motoras da Doença de Parkinson. Método. Trata-se de uma revisão integrativa de literatura, realizada nas bases de dados Literatura Latino-Americana e do Caribe em Ciências da Saúde (LILACS), Literatura Internacional em Ciências da Saúde (MEDLINE), via Pubmed, Physiotherapy Evidence Database (PEDro) e Scientifc Eletronic Library Online (SciELO), utilizando os descritores Parkinson Disease e Virtual Reality, combinados com operador booleano AND. Foram incluídos artigos completos, publicados entre 2012 a 2020 e disponíveis no idioma inglês, sendo excluídos os artigos de revisões, teses, artigos duplicados e os estudos que não abordavam a utilização da realidade virtual na reabilitação da DP. Após a aplicação dos critérios de elegibilidade, foram selecionados 12 artigos. Resultados. A partir dos estudos incluídos, pôde-se observar que a realidade virtual, através de jogos comerciais e personalizados, contribui positivamente para um melhor desempenho motor dos pacientes com DP, sendo utilizada no tratamento dos padrões anormais da marcha, nas alterações na mobilidade funcional, nos déficits de equilíbrio, nos distúrbios da função motora e para o aumento da força muscular desses indivíduos. Conclusão. A realidade virtual é uma ferramenta potencialmente eficaz no processo de reabilitação das alterações motoras dos pacientes com DP, apresentando, em alguns casos, resultados superiores ou igualmente satisfatórios aos das intervenções fisioterapêuticas tradicionais.
\end{abstract}

Unitermos. Doença de Parkinson; Realidade Virtual; Fisioterapia

\footnotetext{
Abstract

Introduction. Virtual reality is a technological tool used in the physiotherapeutic rehabilitation protocol, being a treatment option for individuals affected by Parkinson's Disease (PD). Objective. To address the contributions of virtual reality in the rehabilitation of motor disorders in Parkinson's Disease. Method: This is an integrative literature review, carried out in the Latin American and Caribbean Literature in Health Sciences (LILACS), International Literature in Health Sciences (MEDLINE) databases, via Pubmed, Physiotherapy Evidence Database (PEDro) and Scientifc Electronic Library Online (SciELO), using the descriptors Parkinson Disease and Virtual Reality, combined with the Boolean AND operator. Full articles published between 2012 and 2020 and available in English were included, excluding review
} 
articles, theses, duplicate articles and studies that did not address the use of virtual reality in the rehabilitation of PD. After applying the eligibility criteria, 12 articles were selected. Results. From the included studies, it was observed that virtual reality, through commercial and personalized games, contributes positively to a better motor performance of patients with $P D$, being used in the treatment of abnormal gait patterns, in changes in mobility function, balance deficits, motor function disorders and to increase muscle strength in these individuals. Conclusion. Virtual reality is a potentially effective tool in the process of rehabilitation of motor disorders in patients with PD, presenting, in some cases, results that are superior or equally satisfactory to those of traditional physical therapy interventions.

Keywords. Parkinson's disease; Virtual reality; Physical Therapy

\section{Resumen}

Introducción. La realidad virtual es una herramienta tecnológica utilizada en el protocolo de rehabilitación fisioterapéutica, siendo una opción de tratamiento para las personas afectadas por la Enfermedad de Parkinson (EP). Objetivo. Abordar las contribuciones de la realidad virtual en la rehabilitación de los trastornos motores en la enfermedad de Parkinson. Método. Se trata de una revisión integradora de la literatura, realizada en las bases de datos de Literatura Latinoamericana y del Caribe en Ciencias de la Salud (LILACS), Literatura Internacional en Ciencias de la Salud (MEDLINE), vía Pubmed, Physiotherapy Evidence Database (PEDro) y Scientifc Electronic Library Online (SciELO), utilizando los descriptores Enfermedad de Parkinson y Realidad virtual, combinados con el operador booleano AND. Se incluyeron artículos completos publicados entre 2012 y 2020 y disponibles en inglés, excluyendo artículos de revisión, tesis, artículos duplicados y estudios que no abordaran el uso de la realidad virtual en la rehabilitación de la EP. Después de aplicar los criterios de elegibilidad, se seleccionaron 12 artículos. Resultados. A partir de los estudios incluidos, se pudo observar que la realidad virtual, a través de juegos comerciales y personalizados, contribuye positivamente a un mejor desempeño motor de los pacientes con EP, siendo utilizada en el tratamiento de patrones anormales de marcha, en cambios en la función de movilidad, equilibrio déficits, trastornos de la función motora y para aumentar la fuerza muscular en estos individuos. Conclusión. La realidad virtual es una herramienta potencialmente eficaz en el proceso de rehabilitación de los trastornos motores en pacientes con EP, presentando, en algunos casos, resultados superiores o igualmente satisfactorios a los de las intervenciones de fisioterapia tradicional.

Palabras clave. Enfermedad de Parkinson; Realidad virtual; fisioterapia

Trabalho realizado no Centro Universitário de Ciências e Tecnologia do Maranhão - UNIFACEMA. CaxiasMA, Brasil.

\section{INTRODUÇÃO}

\section{A Doença de Parkinson (DP) é uma doença neurológica,} degenerativa e de caráter progressivo, ocasionada pela perda de neurônios dopaminérgicos situados na substância negra do mesencéfalo ${ }^{1}$. Globalmente, a DP afeta cerca de 10 a 14 pessoas por 100.000 ao ano, o que lhe garante o 
segundo lugar entre as afecções neurodegenerativas mais frequentes no mundo ${ }^{2-3}$.

A etiologia da DP não foi totalmente elucidada, mas acredita-se na participação de mecanismos multifatoriais, entre eles a associação da predisposição genética com a exposição a fatores tóxicos ambientais ${ }^{4}$. Clinicamente, a doença é caracterizada pela presença de sintomas motores e não motores. Os motores são considerados os sintomas típicos, sendo compostos pelos sinais cardinais de rigidez, bradicinesia, tremor em repouso e instabilidade postural. Os não motores compreendem diversas manifestações como os declínios cognitivos, a depressão e a ansiedade ${ }^{5}$.

O manejo terapêutico desse quadro clínico, inclui a terapia medicamentosa, onde é prescrito a reposição de dopamina, intervenções cirúrgicas e fisioterapia6,7.0 tratamento fisioterapêutico tem mostrado, através de inúmeros estudos, seus benefícios na capacidade funcional, no equilíbrio, na força muscular e na marcha desses pacientes ${ }^{8-11}$.

Diversos recursos podem ser empregados como ferramenta terapêutica na fisioterapia, entre eles a realidade virtual (RV). A RV é uma tecnologia computadorizada que proporciona a interação do usuário com a máquina em um ambiente virtual ${ }^{12}$. Na reabilitação da DP à RV vem sendo utilizada, principalmente, nos tratamentos dos distúrbios motores, como nos déficits de equilíbrio ${ }^{13}$.

Diante desse cenário, é essencial compreender os efeitos terapêuticos proporcionados por esse recurso no 
tratamento das alterações motoras dos indivíduos com DP, tendo como finalidade fomentar a prática baseada em evidências na reabilitação desses pacientes. Nessa perspectiva, o presente estudo teve como objetivo abordar as contribuições da realidade virtual na reabilitação das alterações motoras da Doença de Parkinson.

\section{MÉTODO}

Trata-se de uma revisão integrativa de literatura, do tipo exploratória e descritiva. Esta abordagem metodológica é um dos mais amplos métodos de revisão, uma vez que utiliza estudos experimentais e não-experimentais para uma compreensão mais ampla da temática em análise. A construção dessa revisão seguiu as seguintes etapas metodológicas: 1) elaboração da questão norteadora; 2) busca na literatura; 3) categorização dos estudos; 4) avaliação dos estudos incluídos na revisão integrativa; 5) interpretação dos resultados e 6) síntese do conhecimento ${ }^{14}$.

Na primeira etapa foi formulada a questão norteadora, onde originou-se a seguinte questão central da pesquisa: "Quais as contribuições da utilização da realidade virtual na reabilitação das alterações motoras da Doença de Parkinson?".

Com o intuito de responder a referida problemática foi realizado um levantamento de artigos nas bases de dados Literatura Latino-Americana e do Caribe em Ciências da Saúde (LILACS), Literatura Internacional em Ciências da Saúde (MEDLINE), via Pubmed, Physiotherapy Evidence 
Database (PEDro) e Scientifc Eletronic Library Online(SciELO), utilizando os seguintes Descritores em Ciência da Saúde (DeCS) e Medical Subject Headings (MeSH): Parkinson Disease; Virtual Reality. Esses descritores foram combinados utilizando 0 operador booleano $A N D$. O processo de busca foi realizado por dois pesquisadores, de forma independente, nos meses de agosto a setembro de 2020 .

Foram incluídos artigos completos, publicados entre 2012 a 2020 e disponíveis no idioma inglês. Os artigos de revisões, teses, artigos duplicados e os estudos que não abordavam a utilização da realidade virtual na reabilitação da DP foram excluídos.

A categorização dos estudos foi realizada através da matriz de síntese, elaborada no software Microsoft Excel ${ }^{\circledR}$, versão 2019, contendo os seguintes itens: autor e ano; tipo de estudo/nível de evidência; instrumento de avaliação, descrição da intervenção e principais resultados. Para avaliação do nível de evidência científica foi utilizado a classificação do Fineout-Overholt et al. ${ }^{15}$, que permite classificar os artigos em ordem decrescente de evidência, sendo elas: (I) revisão sistemática ou metanálise; (II) ensaios clínicos randomizados; (III) ensaios clínicos sem randomização; (IV) estudos de coorte e de caso-controle; (V) revisão sistemática de estudos descritivos e qualitativos; (VI) único estudo descritivo ou qualitativo e (VII) opinião ou consenso ${ }^{13}$. Os artigos classificados em nível de evidência II 
e III tiveram sua qualidade metodológica avaliada pela escala PEDro.

A escala PEDro, desenvolvida pela Physiotherapy Evidence Database, é uma das mais utilizadas para avaliação da qualidade metodológica de ensaios controlados aleatorizados ou quase-aleatorizados na área da reabilitação. Este instrumento é composto por 11 itens, cada item, com exceção do primeiro, pode pontuar 0 (ausência do indicador) ou 1 (presença do indicador), perfazendo uma pontuação final que varia de 0 a $10^{16}$. Neste estudo, os artigos pertencentes a PEDro tiveram seus escores obtidos na própria base de dados, enquanto os estudos provenientes de outras bases de dados foram avaliados por dois pesquisadores de forma independente.

Por fim, as informações dos artigos foram sintetizadas e analisadas de forma crítica e detalhada, buscando fazer comparações e conclusões com bibliografias atualizadas sobre a temática.

\section{RESULTADOS}

Foram identificados, mediante ao cruzamento dos descritores, 226 artigos. Destes, foram excluídos 24 por estarem repetidos em mais de uma base de dados, resultando em 202 artigos para leitura de títulos e resumos. Após essa etapa, baseado nos critérios de elegibilidade, outros 166 artigos foram excluídos. Desse modo, foram selecionados 36 artigos para a leitura na íntegra. Depois da leitura completa, foram escolhidos 12 artigos. 0 processo de 
busca e seleção dos artigos está representado no fluxograma esquemático da Figura 1.

Figura 1: Fluxograma das etapas de busca e seleção dos artigos analisados.

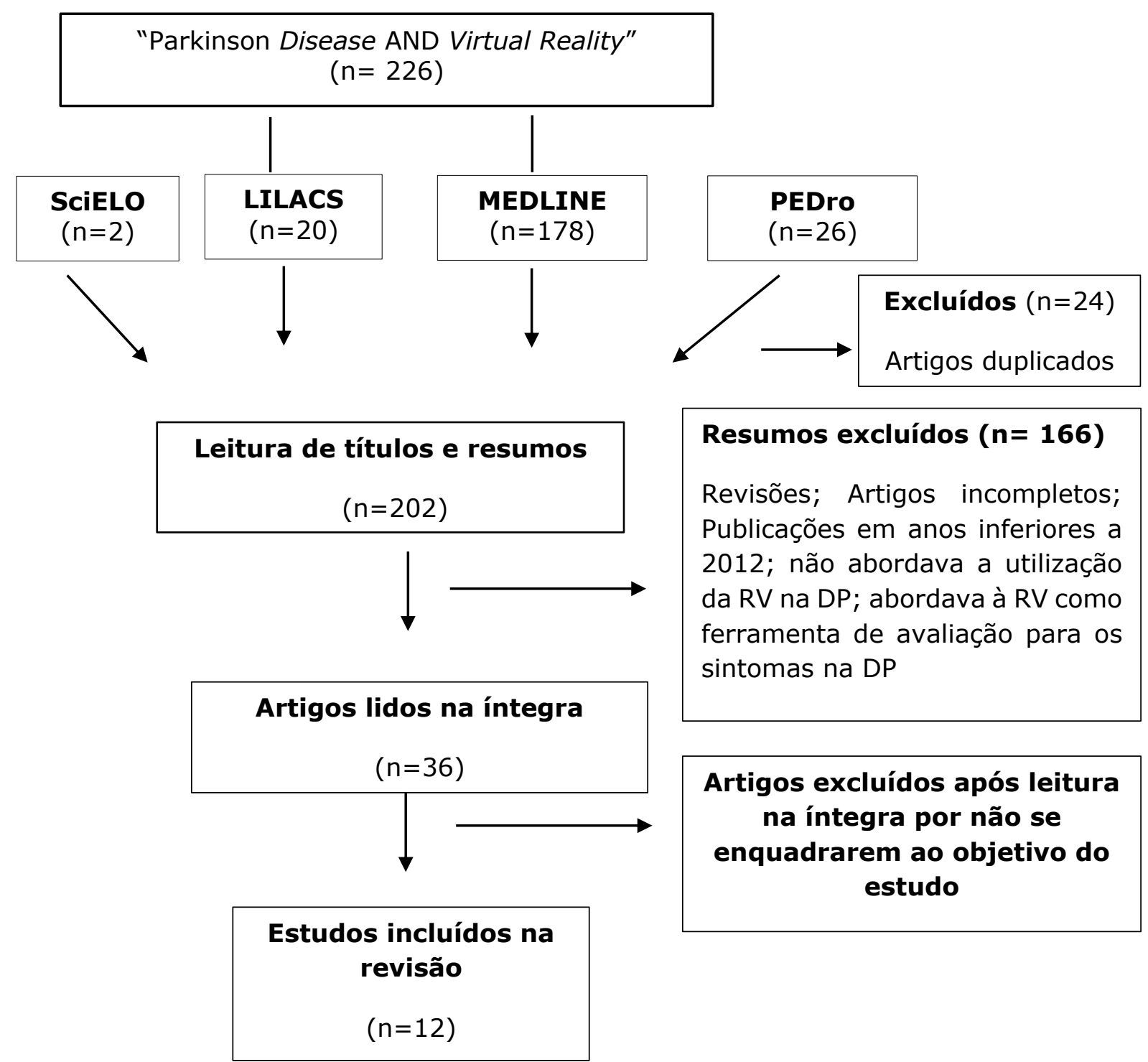

\section{Características dos estudos incluídos}

Ao caracterizar os estudos segundo ao ano de publicação, observou-se uma prevalência no ano de $2018^{17-}$ 19 e $2019^{20-22}$, com três (25\%) estudos em cada ano, seguido 
por $2015^{23,24}$, com duas $(16,7 \%)$ publicações. Nos anos de $2012^{25}, 2014^{26}, 2016^{27}$ e $2020^{28}$ foi constatado um $(8,3 \%)$ artigo para cada ano. Quanto ao tipo de estudo, percebeuse que sete $(58,3 \%)^{20-24,27,28}$ eram ensaio clínico controlado randomizado e três $(25 \%)^{18,25,26}$ estudo piloto. Os demais estudos eram ensaio clínico não randomizado ${ }^{19}$ e de coorte ${ }^{17}$, sendo que cada um desses estudos possuía apenas um (8,3\%) artigo. Em relação ao nível de evidência, constatouse que a maioria dos estudos são classificados no nível II de evidência científica $(n=7 ; 58,3 \%)^{20-24,27,28}$, seguido pelo nível de evidência VI $(n=3 ; 25)^{18,25,26}$, IV $(n=1 ; 8,3 \%)^{17}$ e III $(\mathrm{n}=1 ; 8,3 \%)^{19}$ (Quadro 1 ).

A qualidade dos ensaios clínicos incluídos, de acordo com a escala PEDro, variou de 4 a 7 , sendo que cinco artigos $^{21-24,27}$ pontuaram 7 e os demais obtiveram escores 6 $(n=1)^{19}, 5(n=1)^{28}$ e $4(n=1)^{20}$, evidenciando uma qualidade metodológica de baixa a intermediária (Quadro 2).

Em relação aos resultados da intervenção com RV nas alterações motoras dos pacientes com DP, observou-se que os estudos incluídos avaliaram os efeitos da RV no tratamento dos distúrbios relacionados a marcha, mobilidade funcional, equilíbrio, função motora e força muscular.

Os efeitos do tratamento com RV na marcha foi avaliado em oito artigos $18,19,21-24,26,28$, por meio dos parâmetros cinemáticos da marcha ${ }^{18,23}$ e/ou pelas escalas Functional Gait Assessment (FGA) 21,24 , Dynamic Gait Index (DGI) 22,26,28, Escala de Congelamento da Marcha (FOG) ${ }^{18}$, Escala Clínica de Marcha e Equilíbrio (GABS) ${ }^{18}$, Teste de Caminhada de 10 
metros e Teste de Caminhada de 30 segundos $^{19}$. Todos os estudos demonstraram que a RV contribuiu positivamente para o melhor desempenho da marcha dos participantes (Quadro 1).

A mobilidade funcional dos pacientes com DP após a utilização da RV foi analisada em seis estudos ${ }^{17,21-23,25,27}$, através do teste de Time Up and Go (TUG). Destes, cinco $(83,3 \%)$ artigos ${ }^{17,21-23,27}$ relataram melhora na execução do teste (Quadro 1).

Oito artigos investigaram os efeitos da RV no equilíbrio 17,21-23,25-28. As ferramentas utilizadas para avaliar os resultados da intervenção foram variadas, mas houve predomínio da Escala de Equilíbrio de Berg, que foi usada em cinco estudos ${ }^{17,21,22,25,28}$. Todas as publicações demonstraram que a RV propiciou melhoras na pontuação dos instrumentos de avaliação utilizados, evidenciando uma melhora nos déficits de equilíbrio após a intervenção (Quadro 1).

A eficácia do treinamento com RV na função motora foi avaliada em dois estudos $20-21$. Ambas as pesquisas, obtiveram uma diminuição nos escores da Escala Unificada de Avaliação para Doença de Parkinson (UPDRS III), evidenciando uma redução do comprometimento motor após a intervenção (Quadro 1).

A força muscular, após a intervenção com RV, foi avaliada em um único estudo ${ }^{24}$ entre os artigos incluídos. Os resultados mostraram que a RV foi eficaz para 0 fortalecimento dos músculos flexores/extensores do quadril, 
flexores/extensores do joelho e dorsiflexores/flexores plantar do tornozelo. A força foi avaliada usando um dinamômetro (Quadro 1).

\section{DISCUSSÃO}

O presente estudo teve como objetivo abordar as contribuições da realidade virtual na reabilitação das alterações motoras da Doença de Parkinson. Os resultados dos estudos incluídos sugerem que a RV é um importante recurso terapêutico na reabilitação das alterações motoras dos pacientes com DP, destacando-se no tratamento dos distúrbios relacionados a marcha, mobilidade funcional, equilíbrio, função motora e força muscular desses indivíduos.

A maioria dos estudos incluídos nesta revisão são ensaios clínicos controlados, apresentando, por meio da escala PEDro, uma qualidade metodológica de baixa a intermediária. Contudo, ressalta-se que também foram incluídos estudos piloto e de coorte.

\section{Marcha}

Os efeitos benéficos da RV na marcha foram demostrados em um ensaio clínico controlado randomizado, no qual os 25 pacientes submetidos a dezesseis atendimentos com RV não imersiva, por meio do sistema Nirvana, apresentaram uma melhora na caminhada. Este resultado positivo foi evidenciado pelo aumento da pontuação da escala $\mathrm{DGI}^{28}$. Resultados satisfatórios nos escores da DGI após a intervenção com RV também foram 


\section{encontrados em um outro ensaio clínico controlado}

randomizado ${ }^{22}$ e em um estudo piloto $^{26}$ realizados com

pacientes com DP.

Quadro 1. Sinopse dos dados dos estudos incluídos na revisão de literatura.

\begin{tabular}{|c|c|c|c|}
\hline $\begin{array}{l}\text { Autor, } \\
\text { Ano }\end{array}$ & $\begin{array}{c}\text { Tipo de } \\
\text { estudo/ } \\
\text { Nível de } \\
\text { evidência } \\
\end{array}$ & $\begin{array}{c}\text { Instrumento de } \\
\text { avaliação }\end{array}$ & $\begin{array}{c}\text { Descrição da intervenção e } \\
\text { principais resultados }\end{array}$ \\
\hline $\begin{array}{c}\text { Loureiro } \\
2012^{25}\end{array}$ & Estudo piloto / VI & $\begin{array}{c}\text { Escala de Equilíbrio } \\
\text { Funcional de Berg; TUG; } \\
\text { Alcance funcional. }\end{array}$ & $\begin{array}{l}\text { Pacientes de ambos os sexos com diagnóstico de } \\
\text { DP }(n=6) \text {, submetidos a intervenção com jogos de } \\
\text { Wii Fit da Nintendo Wii, por um período de cinco } \\
\text { semanas, demonstraram melhorias no equilíbrio e } \\
\text { no alcance funcional direito e esquerdo. Os } \\
\text { pacientes não apresentaram alterações } \\
\text { significativas na mobilidade funcional. }\end{array}$ \\
\hline $\begin{array}{l}\text { Severiano } \\
2018^{17}\end{array}$ & $\begin{array}{c}\text { Estudo de Coorte/ } \\
\text { IV }\end{array}$ & $\begin{array}{c}\text { Escala de equilíbrio de } \\
\text { Berg e TUG. }\end{array}$ & $\begin{array}{l}\text { Pacientes de ambos os sexos com diagnóstico de } \\
\text { DP }(n=16) \text {, submetidos ao protocolo de } 20 \\
\text { atendimentos com quatro jogos da Nintendo Wii, } \\
\text { apresentaram melhorias na mobilidade dos } \\
\text { membros inferiores e no equilíbrio. }\end{array}$ \\
\hline $\begin{array}{l}\text { Yang } \\
2016^{27}\end{array}$ & $\begin{array}{l}\text { Ensaio clínico } \\
\text { controlado } \\
\text { randomizado/ II }\end{array}$ & DGI e TUG. & $\begin{array}{l}\text { Pacientes com diagnóstico de } \mathrm{DP}(\mathrm{n}=20) \text {, foram } \\
\text { divididos em grupo experimental (GE) e grupo } \\
\text { controle (GC). O GE recebeu o treinamento de } \\
\text { equilíbrio através de um sistema de RV } \\
\text { desenvolvido pelo Centro de Ciclismo e Saúde de } \\
\text { Taichung, em Taiwan. O GC recebeu o treino de } \\
\text { equilíbrio convencional. As duas intervenções } \\
\text { melhoraram o equilíbrio e a mobilidade dos } \\
\text { participantes. }\end{array}$ \\
\hline $\begin{array}{c}\text { Liao } \\
2015^{23}\end{array}$ & $\begin{array}{l}\text { Ensaio clínico } \\
\text { controlado } \\
\text { randomizado / II }\end{array}$ & $\begin{array}{l}\text { Sistema Liberty, sistema } \\
\text { Balance Master e TUG. }\end{array}$ & $\begin{array}{l}\text { Os participantes com diagnóstico de DP }(n=36) \\
\text { foram divididos em } 3 \text { grupos, sendo eles: grupo } \\
\text { controle }(n=12) \text {, receberam educação para a } \\
\text { prevenção de quedas; grupo RVWii }(n=12) \text {, que } \\
\text { receberam o treinamento com Wii Fit e grupo com } \\
\text { exercício tradicional }(n=12) \text {. Os indivíduos do } \\
\text { grupo RVWii apresentaram resultados satisfatórios } \\
\text { no que respeito ao tamanho da passada de } \\
\text { cruzamento e velocidade diante dos obstáculos, } \\
\text { equilíbrio dinâmico e mobilidade funcional. Esses } \\
\text { benefícios foram superiores ao do grupo controle } \\
\text { e semelhantes ao do grupo de exercício } \\
\text { tradicional. }\end{array}$ \\
\hline $\begin{array}{l}\text { Liao } \\
2015^{24}\end{array}$ & $\begin{array}{l}\text { Ensaio clínico } \\
\text { controlado } \\
\text { randomizado / II }\end{array}$ & $\begin{array}{l}\text { FGA e dinamômetro } \\
\text { portátil. }\end{array}$ & $\begin{array}{l}\text { Os participantes foram divididos em grupo } \\
\text { controle( } n=12) \text {, grupo com exercícios tradicionais } \\
\text { ( } n=12) \text { e grupo com RV por Wii Fit }(n=12) \text {. Os } \\
\text { exercícios tradicionais e os com RV apresentaram } \\
\text { resultados igualmente satisfatórios na locomoção } \\
\text { e capacidade de força muscular isométrica dos } \\
\text { músculos flexores/extensores do quadril, } \\
\text { flexores/extensores do joelho } \\
\text { dorsiflexores/flexores plantar do tornozelo. O } \\
\text { grupo controle apresentou resultados inferiores } \\
\text { quando comparados com os dois grupos. }\end{array}$ \\
\hline $\begin{array}{l}\text { Pazzaglia } \\
2020^{28}\end{array}$ & $\begin{array}{l}\text { Ensaio clínico } \\
\text { controlado } \\
\text { randomizado / II }\end{array}$ & $\begin{array}{c}\text { Escala de equilíbrio de } \\
\text { Berg e } \\
\text { DGI. }\end{array}$ & $\begin{array}{l}\text { Os pacientes com DP foram randomizados em } \\
\text { programa de reabilitação convencional }(n=26) \text { e } \\
\text { reabilitação com RV ( } n=25) \text {, durante seis } \\
\text { semanas. No grupo convencional foi feito um } \\
\text { programa com fase de aquecimento, fase ativa e } \\
\text { de resfriamento. No grupo RV os pacientes fizeram } \\
\text { sete tipos de exercícios com o equipamento } \\
\text { Nirvana. Os indivíduos submetidos a terapia com } \\
\text { RV apresentam resultados mais significativos do } \\
\text { que os da reabilitação convencional no que se } \\
\text { refere ao equilíbrio e marcha. }\end{array}$ \\
\hline
\end{tabular}


Quadro 1 (cont.). Sinopse dos dados dos estudos incluídos na revisão de literatura.

\begin{tabular}{|c|c|c|c|}
\hline $\begin{array}{l}\text { Autor, } \\
\text { Ano }\end{array}$ & $\begin{array}{c}\text { Tipo de } \\
\text { estudo/ } \\
\text { Nível de } \\
\text { evidência } \\
\end{array}$ & $\begin{array}{l}\text { Instrumento de } \\
\text { avaliação }\end{array}$ & $\begin{array}{l}\text { Descrição da intervenção e } \\
\text { principais resultados }\end{array}$ \\
\hline $\begin{array}{l}\text { Cikajlo } \\
2019^{20}\end{array}$ & $\begin{array}{l}\text { Ensaio clínico } \\
\text { controlado } \\
\text { randomizado / II }\end{array}$ & UPDRS III & $\begin{array}{l}\text { Os participantes foram divididos através de sorteio } \\
\text { em dois grupos, um submetido à RV não imersiva } \\
(\mathrm{n}=10) \text {, como o uso do laptop e o outro à RV } \\
\text { imersiva( }(n=10) \text {, através do uso de óculos. O } \\
\text { sistema de RV utilizado foi desenvolvido pelos } \\
\text { pesquisadores, o sistema de exergaming } 10 \text { Cubes. } \\
\text { Ambos os protocolos resultaram em efeitos } \\
\text { igualmente positivos na função motora do membro } \\
\text { superior. Entretanto, o grupo 3D aumentou sua } \\
\text { motivação no decorrer da intervenção, enquanto o } \\
\text { 2D foi perdendo gradativamente o entusiasmo. }\end{array}$ \\
\hline $\begin{array}{c}\text { Feng } \\
2019^{21}\end{array}$ & $\begin{array}{l}\text { Ensaio clínico } \\
\text { controlado } \\
\text { randomizado / II }\end{array}$ & $\begin{array}{l}\text { Escala de equilíbrio de } \\
\text { Berg, TUG, UPDRS III e } \\
\text { FGA. }\end{array}$ & $\begin{array}{l}\text { Os pacientes com DP }(\mathrm{n}=28) \text { foram divididos em } \\
\text { grupo controle }(\mathrm{GC}) \text { e grupo experimental }(\mathrm{GE}) \text {. O } \\
\text { GC recebeu a fisioterapia convencional, enquanto } \\
\text { o GE foi submetido a intervenção com três jogos } \\
\text { virtuais. O GE apresentou resultados } \\
\text { significativamente melhores do que o GC no } \\
\text { equilíbrio, na mobilidade, função motora geral e } \\
\text { marcha. }\end{array}$ \\
\hline $\begin{array}{c}\text { Nuic } \\
2018^{18}\end{array}$ & Estudo piloto / VI & $\begin{array}{l}\text { GABS, FOG, parâmetros } \\
\text { cinemáticos da marcha. }\end{array}$ & $\begin{array}{l}\text { Os pacientes com } \mathrm{DP}(\mathrm{n}=10) \text { foram submetidos a } \\
18 \text { sessões de um programa de reabilitação com } \\
\text { um vídeo game personalizado, o Toap Run. Após } \\
\text { a intervenção, houve uma redução no } \\
\text { congelamento de marcha e melhora em alguns } \\
\text { parâmetros cinemáticos, sendo eles: aumento do } \\
\text { comprimento do passo e da velocidade da marcha } \\
\text { e diminuição do tempo de apoio duplo. }\end{array}$ \\
\hline $\begin{array}{l}\text { Alves } \\
2018^{19}\end{array}$ & $\begin{array}{l}\text { Ensaio clínico não } \\
\text { randomizado / III }\end{array}$ & $\begin{array}{l}\text { Teste de caminhada de } \\
10 \text { metros e teste de } \\
\text { caminhada de } 30 \\
\text { segundos. }\end{array}$ & $\begin{array}{l}\text { Os participantes foram divididos em três grupos de } \\
\text { nove pessoas cada, sendo eles: grupo controle, } \\
\text { que não recebeu nenhum tratamento; grupo Wii, } \\
\text { foi submetido a } 4 \text { jogos Nintendo Wii; grupo Xbox } \\
\text { Kinect, realizou uma intervenção com } 4 \text { jogos do } \\
\text { Xbox Kinect. Os pacientes submetidos à RV com } \\
\text { Nintendo Wii apresentaram um aumento do } \\
\text { comprimento da passada e da distância } \\
\text { percorrida. O grupo controle o o submetido ao jogo } \\
\text { de Xbox Kinect não apresentaram melhorias em } \\
\text { nenhum aspecto avaliado. }\end{array}$ \\
\hline $\begin{array}{l}\text { Santos } \\
2019^{22}\end{array}$ & $\begin{array}{l}\text { Ensaio clínico } \\
\text { controlado } \\
\text { randomizado / II }\end{array}$ & $\begin{array}{l}\text { Escala de Equilíbrio de } \\
\text { Berg, DGI e TUG. }\end{array}$ & $\begin{array}{l}\text { Os indivíduos foram divididos em três grupos, } \\
\text { sendo eles: grupo que recebeu o tratamento com } \\
\text { Nintendo Wii ( } \mathrm{n}=13) ; \text { grupo que realizou } \\
\text { exercícios convencionais ( } \mathrm{n}=14) ; \text { grupo que } \\
\text { recebeu a combinação das duas intervenções } \\
(\mathrm{n}=14) \text {. Todas as intervenções provocaram } \\
\text { melhoras no equilíbrio, marcha e mobilidade } \\
\text { funcional dos pacientes com DP, não havendo } \\
\text { diferença estatística entre os grupos. }\end{array}$ \\
\hline $\begin{array}{l}\text { Pompeu } \\
2014^{26}\end{array}$ & Estudo piloto / VI & $\begin{array}{c}\text { Teste de equilíbrio } \\
\text { balance evaluation } \\
\text { systems test (BESTes) e } \\
\text { DGI. }\end{array}$ & $\begin{array}{l}\text { Os participantes com DP }(n=7) \text { foram submetidos } \\
\text { a um protocolo de RV com quatro jogos do Kinect. } \\
\text { A intervenção com Kinect melhorou a o equilíbrio } \\
\text { e a marcha desses indivíduos. }\end{array}$ \\
\hline
\end{tabular}


Quadro 2. Avaliação da qualidade metodológica dos ensaios clínicos incluídos na revisão, por meio da escala PEDro.

\begin{tabular}{|c|c|c|c|c|c|c|c|c|c|c|c|c|}
\hline Autor, ano & $\mathbf{1}$ & $\mathbf{2}$ & $\mathbf{3}$ & $\mathbf{4}$ & $\mathbf{5}$ & $\mathbf{6}$ & $\mathbf{7}$ & $\mathbf{8}$ & $\mathbf{9}$ & $\mathbf{1 0}$ & $\mathbf{1 1}$ & Total \\
\hline Yang 201627 & - & 1 & 0 & 1 & 0 & 0 & 1 & 1 & 1 & 1 & 1 & 7 \\
\hline Liao 201523 & - & 1 & 1 & 1 & 0 & 0 & 1 & 1 & 0 & 1 & 1 & 7 \\
\hline Liao 201524 & - & 1 & 1 & 1 & 0 & 0 & 1 & 1 & 0 & 1 & 1 & 7 \\
\hline Pazzaglia 202028 & - & 1 & 0 & 1 & 0 & 0 & 1 & 0 & 0 & 1 & 1 & 5 \\
\hline Cikajlo 201920 & - & 1 & 0 & 0 & 0 & 0 & 0 & 1 & 0 & 1 & 1 & 4 \\
\hline Feng 201921 & - & 1 & 0 & 1 & 0 & 0 & 1 & 1 & 1 & 1 & 1 & 7 \\
\hline Alves 2018 19 & - & 0 & 0 & 1 & 0 & 0 & 1 & 1 & 1 & 1 & 1 & 6 \\
\hline Santos 201922 & - & 1 & 0 & 1 & 0 & 0 & 1 & 1 & 1 & 1 & 1 & 7 \\
\hline
\end{tabular}

Ratificando esses dados, um ensaio clínico controlado randomizado, realizado com 36 indivíduos com DP, demonstrou que os noves participantes submetidos a intervenção com Wii Fit, realizada duas vezes por semana por um período de seis semanas, melhoraram 0 comprimento da passada de cruzamento e a velocidade diante dos obstáculos 23 .

Corroborando com esses achados, um estudo piloto realizado em Paris, França, com 10 pacientes diagnosticados com DP, revelou, depois de dezoito sessões com um vídeo game personalizado, um aumento no comprimento do passo e na velocidade da marcha, diminuição do tempo de apoio duplo e redução na pontuação da escala de congelamento da marcha dos participantes, demonstrando que essa ferramenta afetou positivamente os parâmetros de iniciação da marcha e o desempenho na caminhada ${ }^{18}$.

Outro estudo, um ensaio clínico não randomizado, realizado em Brasília, Brasil, com 27 participantes com DP, 
demonstrou que o grupo de nove pessoas submetidas a quatro jogos da Nintendo Wii obtiveram um aumento significativo na distância percorrida e no comprimento da passada durante o teste de caminhada de 30 segundos, mesmo durante a atividade de dupla tarefa ${ }^{19}$.

Quando os efeitos terapêuticos da RV sobre a marcha foram comparados com os gerados pela fisioterapia convencional, dois estudos, um ensaio clínico controlado randomizado ${ }^{21}$ e um estudo piloto ${ }^{26}$, demonstraram que a RV proporcionou resultados superiores aos das intervenções tradicionais. Em oposição a esses achados, dois ensaios clínicos controlados randomizados ${ }^{22,24}$ evidenciaram que a RV promove efeitos terapêuticos igualmente satisfatórios aos da reabilitação fisioterapêutica convencional.

Uma hipótese para explicar os efeitos positivos da RV nos distúrbios da marcha é que os exercícios usados podem ter contribuído para aprendizagem e reaprendizagem motora e para o aumento da força muscular, que refletiu na caminhada dos pacientes com DP22.

\section{Mobilidade funcional}

Quatro ensaios clínicos controlados randomizados $21-23,27$ e um estudo de coorte ${ }^{17}$ demostraram, através da redução no tempo de execução do TUG, uma melhora da mobilidade funcional dos pacientes que foram submetidos a intervenção com RV. De encontro a esse achado, um estudo piloto realizado com 6 participantes com DP, em Curitiba, Brasil, 
não demonstrou alterações no tempo de execução do TUG após o tratamento com Wii Fit da Nintendo Wii ${ }^{25}$.

Uma mobilidade funcional adequada proporciona uma maior independência dos pacientes com DP, e, consequentemente, reduz a sobrecarga dos seus cuidadores $^{21}$. Os resultados positivos na mobilidade podem ser atribuídos aos efeitos benéficos da RV no equilíbrio e na integração sensorial. O equilíbrio preservado permite que os indivíduos se movimentem com uma velocidade e padrões corretos, enquanto a integração sensorial garante que as alterações no posicionamento e ambiente sejam interpretadas ${ }^{23}$.

\section{Equilíbrio}

A instabilidade postural é um dos sintomas motores mais incapacitantes da DP, tendo em vista que aumenta a propensão de quedas e consequentemente a morbidade ${ }^{28}$. Neste estudo, os resultados evidenciaram que os pacientes com DP submetidos ao tratamento com RV obtiveram efeitos benéficos no equilíbrio estático e dinâmico ${ }^{17,21-23,25-28 . ~}$

Um estudo piloto, realizado com 6 pacientes com DP, revelou que houve uma melhora no desempenho da Escala de Equilíbrio de Berg e no alcance funcional direito e esquerdo após a intervenção com Wii Fit $^{25}$. Corroborando com esses achados, um ensaio clínico controlado randomizado, realizado com 36 pacientes com DP, em Taiwan, mostrou que o grupo de 9 participantes que receberam o treinamento com Wii Fit melhoraram a 
velocidade de movimento, excursão máxima e o controle direcional durante o equilíbrio dinâmico ${ }^{23}$.

Quando os efeitos da RV no equilíbrio foram comparados com os proporcionados pelo tratamento fisioterapêutico tradicional, um ensaio clínico controlado randomizado relatou que os 10 participantes que receberam o treinamento com um sistema virtual de equilíbrio, desenvolvido em Taiwan, obtiveram efeitos igualmente satisfatórios ao do grupo que foi submetido ao tratamento com os exercícios convencionais ${ }^{27}$. Resultados semelhantes foram encontrados em um outro ensaio clínico controlado randomizado, que demonstrou que a intervenção com Nintendo Wii foi tão eficaz para o equilíbrio dos pacientes com DP quanto os exercícios tradicionais isolados ou combinados com a Nintendo Wiir2 .

Em oposição a esses achados, dois ensaios clínicos controlados randomizados, mostraram que a intervenção com RV foi mais eficiente do que a fisioterapia convencional para o tratamento dos déficits de equilíbrio dos pacientes com $\mathrm{DP}^{21,28}$. Os resultados superiores propiciados pela RV podem ser atribuídos ao fato desta ferramenta estimular as funções motoras e cognitivas simultaneamente, fornecer feedback visual e auditivo do desempenho ${ }^{21,28}$, além de estimular a neuroplasticidade ${ }^{17,26}$.

\section{Função motora}

Um ensaio clínico controlado randomizado, realizado na Eslovênia, com 20 participantes com DP, evidenciou que 
tanto os pacientes submetidos à RV imersiva $(n=10)$ quanto os que receberam a RV não imersiva $(n=10)$ de um sistema personalizado, obtiveram, após dez atendimentos, resultados igualmente satisfatórios na pontuação da UPDR III relacionada a função do membro superior (MS), ou seja, os movimentos e os tremores das mãos, pronação e supinação do antebraço ${ }^{20}$. Os resultados positivos proporcionados pela RV no membro superior são extremamente relevantes para um melhor desempenho das atividades de vida diária (AVD's) dos pacientes com DP, tendo em vista que grandes partes delas exigem coordenação dos movimentos dos membros superiores, manipulação e preensão de objetos ${ }^{20}$.

Esses achados corroboram com o de outro estudo, um ensaio clínico controlado randomizado, que ao comparar os efeitos da RV com o dos exercícios tradicionais na função motora geral dos indivíduos com DP, identificou que o treinamento com RV foi mais eficaz para melhorar o desempenho motor desses pacientes. A razão da superioridade dessa ferramenta diante da fisioterapia convencional foi atribuída aos diversos estímulos gerados pela RV, que reduzem os espasmos musculares e aumentam a sensibilidade dos órgãos auditivos, auxiliando nos ajustes posturais diante de perturbações ${ }^{21}$.

\section{Força muscular}

A eficácia do treinamento com RV na força muscular foi demonstrada em um ensaio clínico controlado randomizado, 
no qual os 12 indivíduos com DP submetidos ao tratamento com Wii Fit, por um período de seis semanas, obtiveram um aumento da força muscular isométrica dos músculos flexores/extensores do quadril, flexores/extensores do joelho e dorsiflexores/flexores plantar do tornozelo. 0 fortalecimento gerado pela RV obedece aos mesmos princípios dos exercícios tradicionais ${ }^{24}$.

Os efeitos positivos da RV na força muscular também foram evidenciados em outras pesquisas ${ }^{29,30}$. Um estudo piloto realizado na Coreia verificou que 18 idosos submetidos ao jogo da Xbox 360 da Kinect, o Your Shape Fitness Evolved, por um período de oito semanas, apresentaram melhoras na força muscular dos músculos flexores, extensores, adutores e abdutores do quadril ${ }^{29}$. Outro estudo, um ensaio clínico controlado randomizado, relatou que nove crianças com Paralisia cerebral submetidas ao tratamento com programa de jogging do Nintendo Wii Fit Plus, apresentaram um aumento da força muscular do quadríceps e isquiotibiais ${ }^{30}$.

Destaca-se que o presente estudo apresenta algumas limitações que devem ser consideradas. Primeiro, os estudos incluídos possuem abordagens metodológicas distintas, 0 que dificulta a comparação dos resultados. Segundo os ensaios clínicos incluídos apresentam qualidade metodológica de baixa a intermediária. Terceiro, abordar as contribuições da RV em todas as alterações motoras da DP impediram que os protocolos utilizados para proporcionar os benéficos fossem mais relatados e discutidos. 


\section{CONCLUSÃO}

Os estudos incluídos nessa revisão integrativa evidenciaram que a RV, através de jogos personalizados e comerciais, especialmente o jogo Wii Fit, é uma opção potencialmente eficaz para o tratamento fisioterapêutico das alterações motoras dos pacientes com DP. Os sintomas motores que apresentaram melhorias após a intervenção foram os relacionados a marcha, mobilidade funcional, equilíbrio, função motora e força muscular. Quando esses efeitos foram comparados com os gerados pela fisioterapia tradicional, observou-se que a RV proporcionou resultados igualmente satisfatórios e/ou superiores no tratamento dos distúrbios da marcha, equilíbrio e função motora geral.

Contudo, ressalta-se que ainda é necessário o desenvolvimento de novas pesquisas que abordem o número de repetições, a duração e a frequência da intervenção mais adequados para propiciar tais efeitos terapêuticos da RV nas alterações motoras da DP.

\section{REFERÊNCIAS}

1. Ranchet M, Morganc JC, Akinwuntand AE, Devos H. Visual search and target detection during simulated driving in Parkinson's disease. Accid Anal Prev $2020 ; 134: 105328$.

https://doi.org/10.1016/j.aap.2019.105328

2. Ferreira FD, Ferreira FMD, Heleno RB, Mella Junior SE. Doença de Parkinson: Aspectos Fisiopatológicos e Terapêuticos. Rev Saúde Pesq 2010;3:221-8.

https://periodicos.unicesumar.edu.br/index.php/saudpesq/article/vie w/1353/1080

3.Guerdão MDQP, Silva SMCA, Silva MLN, Roediger MA. Estado nutricional e ingestão proteica de idosos com doença de Parki nson. Rev Eletr Acervo Saúde 2019;11:1-10. https://doi.org/10.25248/reas.e219.2019

4. Toro R, Downward GS, Mark MVD, Brouwer M, Huss A, Peters $S$, et al. Parkinson's disease and long-term exposure to outdoor air pollution: 
A matched case-control study in the Netherlands. Environ Int 2019;129:28-34. https://doi.org/10.1016/j.envint.2019.04.069

5.Christofoletti G, Cândido ER, Olmedo L, Miziara SRB, Beinotti F. Efeito de uma intervenção cognitivo-motora sobre os sintomas depressivos de pacientes com doença de Parkinson. J Bras Psiquiatr 2012;61:78-83. https://doi.org/10.1590/S0047-20852012000200004 6. Ferrazzoli D, Ortelli P, Cucca A, Bakdounes L, Canesi M, Volpe D. Motor-cognitive approach and aerobic training: a synergism for rehabilitative intervention in Parkinson's disease. Neurodegener Dis Manag 2020;10:41-55. https://doi.org/10.2217/nmt-2019-0025

7.Pessoa RR, Moro A, Munhoz RP, Teive HAG, Lees AJ. Apomorphine in the treatment of Parkinson's disease: a review. Arq Neuropsiquiatr 2018;76:840-8. https://doi.org/10.1590/0004-282x20180140

8. Agosti V, Vitale C, Avella D, Rucco R, Santangelo G, Sorrentino P, et al. Erratum to: Effects of Global Postural Reeducation on gait kinematics in parkinsonian patients: a pilot randomized threedimensional motion analysis study. Neurol Sci 2016;37:515-22. https://doi.org/10.1007/s10072-015-2433-5

9.Bertoldi FC, Silva JAMG, Faganello-Navega FR. Influência do fortalecimento muscular no equilíbrio e qualidade de vida em indivíduos com doença de Parkinson. Fisioter Pesqu 2013;20:117-22. https://doi.org/10.1590/S1809-29502013000200004

10.Stozek J, Rudzińska M, Pustułka-Piwnik U, Szczudlik A. The effect of the rehabilitation program on balance, gait, physical performance and trunk rotation in Parkinson's disease. Aging Clin Exp Res 2016;28:1169-77. https://doi.org/10.1007/s40520-015-0506-1

11.Tramontano M, Bonnì S, Cinnera AM, Marchetti F, Caltagirone C, Koch G. Blindfolded Balance Training in Patients with Parkinson's Disease: A Sensory-Motor Strategy to Improve the Gait. Parkinsons Dis 2016;2016:1-6. https://doi.org/10.1155/2016/7536862

12. Khalil H, Al-Sharman A, El-Salem K, Alghwiri AA, Al-Shorafat D, Khazaaleh $S$, et al. The development and pilot evaluation of virtual reality balance scenarios in people with multiple sclerosis (MS): A feasibility study. NeuroRehabilitation 2018;43:473-82. https://doi.org/10.3233/NRE-182471

13.Freitag F, Brucki SMD, Barbosa AF, Chen J, Souza CO, Valente DF, et al. Is virtual reality beneficial for dual-task gait training in patients with Parkinson's disease? A systematic review. Dement Neuropsicol 2019;13:259-67. $\quad$ https://doi.org/10.1590/1980-57642018dn13030002

14.Souza MTS, Silva MDS, Carvalho R. Revisão integrativa: o que é e como fazer. Einstein 2010;8:102-6. https://doi.org/10.1590/s167945082010 rw1134

15.Fineout-Overholt E, Melnyk BM, Stillwell SB, Williamson K. Evidence-Based Practice Step-by-Step: Critical Appraisal of the Evidence: Part I. Am J Nursing 2010;110:47-52. https://doi.org/10.1097/01.NAJ.0000383935.22721.9c

16.Santos ACP, Fonseca HHV, Xavier SS. Nível de evidência científica de artigos sobre fisioterapia motora em pacientes críticos no brasil: 
revisão integrativa de literatura. BJSCR 2019;26:1-9. https://www. mastereditora.com.br/periodico/20190306 115002.pdf 17.Severiano MIR, Zeigelboim BS, Teive HAG, Santos GJB, Fonseca VR. Effect of virtual reality in Parkinson's disease: a prospective observational study. Arq Neuropsiquiatr 2018;76:78-84. https://doi.org/10.1590/0004-282x20170195

18. Nuic D, Vinti M, Karachi C, Foulon P, Van Hamme A, Welter ML. The feasibility and positive effects of a customised videogame rehabilitation programme for freezing of gait and falls in Parkinson's disease patients: a pilot study. J Neuroeng Rehabil 2018;15:1-11. https://doi.org/10.1186/s12984-018-0375-x

19.Alves MLM, Mesquita BS, Morais WS, Leal JC, Satler CE, Mendes FAS. Nintendo $\mathrm{Wii}^{\mathrm{TM}}$ Versus Xbox Kinect ${ }^{\mathrm{TM}}$ for Assisting People With Parkinson's Disease. Percept Mot Skills 2018;125:546-65. https://doi.org/10.1177/0031512518769204

20.Cikajlo I, Potisk KP. Advantages of using 3D virtual reality-based training in persons with Parkinson's disease: A parallel study. J Neuroeng Rehabil 2019;16:1-14. https://doi.org/10.1186/s12984019-0601-1

21.Feng $\mathrm{H}$, Li C, Liu J, Wang L, Ma J, Li G, et al. Virtual reality rehabilitation versus conventional physical therapy for improving balance and gait in Parkinson's disease patients: A randomized controlled trial. Med Sci Monit 2019;25:4186-92. https://doi.org/10.12659/MSM.916455

22.Santos P, Machado T, Santos L, Ribeiro N, Melo A. Efficacy of the Nintendo Wii combination with Conventional Exercises in the rehabilitation of individuals with Parkinson's disease: A randomized clinical trial. NeuroRehabilitation 2019;45:255-63. https://doi.org/10.3233/NRE-192771

23.Liao YY, Yang YR, Cheng SJ, Wu YR, Fuh JL, Wang RY. Virtual reality-based training to improve obstacle-crossing performance and dynamic balance in patients with Parkinson's disease. Neurorehabil Neural Repair 2015;29:658-67.

https://doi.org/10.1177/1545968314562111

24.Liao YY, Yang YR, Wu YR, Wang RY. Virtual Reality-Based Wii Fit Training in Improving Muscle Strength, Sensory Integration Ability, and Walking Abilities in Patients with Parkinson's Disease: A Randomized Control Trial. Int J Gerontol 2015;9:190-5. https://doi.org/10.1016/j.ijge.2014.06.007

25.Loureiro APC, Ribas CG, Zotz TGG, Chen R, Ribas F. Feasibility of virtual therapy in rehabilitation of Parkinson's disease patients: pilot study. Fisioter Mov 2012;25:659-66. https://doi.org/10.1590/S010351502012000300021

26.Pompeu JE, Arduini LA, Botelho AR, Fonseca MB, Pompeu SM, Torriani-Pasin C, et al. Feasibility, safety and outcomes of playing Kinect Adventures!TM for people with Parkinson's disease: a pilot study. Physiotherapy

2014;100:162-8.

https://doi.org/10.1016/j.physio.2013.10.003 
27.Yang WC, Wang HK, Wu RM, Lo CS, Lin KH. Home-based virtual reality balance training and conventional balance training in Parkinson's disease: A randomized controlled trial. J Formos Med Assoc 2018;115:734-43. https://doi.org/10.1016/j.jfma.2015.07.012

28.Pazzaglia C, Imbimbo I, Tranchita E, Minganti C, Ricciardi D, Lo Monaco $\mathrm{R}$, et al. Comparison of virtual reality rehabilitation and conventional rehabilitation in Parkinson's disease: a randomised controlled trial. Physiotherapy 2020;106:36-42. https://doi.org/10.1016/j.physio.2019.12.007

29.Kim J, Son J, Ko N, Yoon B. Unsupervised virtual reality-based exercise program improves hip muscle strength and balance control in older adults: a pilot study. Arch Phys Med Rehabil 2013;94:937-43. https://doi.org/10.1016/j.apmr.2012.12.010

30.Cho C, Hwang W, Hwang S, Chung Y. Treadmill training with virtual reality improves gait, balance, and muscle strength in children with cerebral palsy. Tohoku J Exp Med 2016;238:213-8. https://doi.org/10.1620/tjem.238.213 